\title{
CLINICAL PROFILE AND OUTCOME OF ACUTE KIDNEY INJURY IN INTENSIVE CARE UNIT OF A TEACHING HOSPITAL
}

Bidhan Shrestha, ${ }^{1 *}$ Sabita Shrestha, ${ }^{2}$ Rakshya Shrestha, ${ }^{2}$ Pramod Paudel, ${ }^{1}$ Hari Krishna Dhakal, ${ }^{1}$ Mukesh Ranjan Shah, ${ }^{1}$ Suresh Kumar Deep, ${ }^{1}$ Sabina Sedhai ${ }^{1}$

${ }^{1}$ Department of Internal Medicine, Chitwan Medical College Teaching Hospital, Bharatpur, Chitwan, Nepal ${ }^{2}$ Department of Obstetrics \& Gynaecology, Chitwan Medical College Teaching Hospital, Bharatpur, Chitwan, Nepal

*Correspondence to: Dr Bidhan Shrestha, Department of Medicine, Chitwan Medical College, Bharatpur, Chitwan, Nepal. Email:bidhansabi@gmail.com.

\begin{abstract}
Objectives: Acute kidney injury is one of the most common cause of hospitalization in developing countries. Causes of AKI are multifactorial. Most of AKI are community acquired. The objective of the study was to identify the clinical profile and outcome of acute kidney disease. Subject and Methodology: 30 patients from Chitwan Medical College outpatient clinic were included in the study from November 2014 to April 2015. A brief history and clinical examinations were taken from all patients along with laboratory tests for Renal function tests, urine output, metabolic parameters and hematological profile. Results: 19 males (63.3\% and 11 females (36.7\%) were studied. The main causes for AKI were sepsis $(46.6 \%)$ followed by hepatic causes $(16.6 \%)$, gastroenteritis $(10 \%)$ and others $(10.2 \%)$. Out of 30 patients, 19 recovered $(63.3 \%)$ and were discharged and $11(36.7 \%)$ died. Most of the deaths were in injury $(37.5 \%)$ and failure (42.8\%) stages of RIFLE criteria. Out of 19 recovered $16(84.21 \%)$ patients did not need any renal replacement therapy whereas $3(\mathbf{1 5 . 8 \% )}$ patients had to undergo hemodialysis. Conclusion: Early identification of kidney injury may lead to lesser renal replacement therapy and better prognosis. However late presentations of AKI have higher hospital mortality rate.
\end{abstract}

Key Words: AKI, ICU, Teaching Hospital

\section{INTRODUCTION}

Acute kidney injury, previously termed as acute renal failure, is a spectrum of disease characterized by abrupt onset of deterioration in kidney function resulting in retention of nitrogenous and other waste products. AKI is a heterogenous group of conditions sharing common features mainly increase in level of serum urea, creatinine and decrease in production of urine. ${ }^{1}$ Many experts tried to classify Acute Kidney Injury, amid controversies, RIFLE system was developed and advocated in 2007. Later in 2012 a larger multidisciplinary group proposed a slight modification in RIFLE criteria called as AKIN (Acute Kidney Injury Network) criteria. ${ }^{2}$ Both these criteria have been proposed to achieve an early diagnosis and prognosis of AKI patients. ${ }^{4}$ One of the most common clinical condition in a hospitalized patient in ICU is AKI the incidence being $2-5 \% .^{3}$ Hospital mortality is directly proportional to the stages of AKI. There has been an increase in the incidence of ICU related AKI during last few decades probably the reason being increased diagnosis of sepsis related admissions, increase prevalence of risk factors for AKI such as Chronic Kidney Disease, Diabetes Mellitus and Congestive heart failure. ${ }^{2}$ Major surgeries, advanced age, liver diseases have also been implicated as risk factors for development of AKI. ${ }^{3}$ AKI is a significant contributor towards morbidity and mortality in ICU patients as supported by the program to improve care in acute renal disease(PICARD) and beginning and ending supportive therapy for kidney (BEST) trials. $^{4}$ Early recognition and management can make AKI reversible but at the same time delay in diagnosis may lead to higher rate of morbidity and mortality. The most common cause for the mortality in AKI are elderly people with complex diseases, sepsis and multi organ failure. AKI is not the cause 
of death however it tells us the depth of underlying disease. Treatment in AKI depends on its early recognition, comprehensive understanding of the clinical spectrum of disease and potential areas of intervention. ${ }^{1}$

We undertook a prospective evaluation in patients with AKI in relation to the most susceptible population, ethnic groups, etiology, outcome and modality of treatment in an Intensive Care Unit of a Teaching Hospital of Chitwan district in Nepal.

\section{METHODOLOGY}

The study was conducted by the Department of Internal Medicine in Chitwan Medical College and teaching hospital, a tertiary centre in Mid Central Nepal, during the period of November 2014 to April 2015. We screened and prospectively studied all the patients who were admitted and fulfilled the inclusion criteria during ICU stay.

Definition: The definition of Acute kidney injury was proposed on the basis of RIFLE criteria in 2002 by Acute Dialysis Initiative which was especially for AKI in critically ill patients. RIFLE includes risk, injury, failure, loss and End stage kidney disease where the first three defined the grades of severity and the last two the outcome classes. Recently in 2007 Acute Kidney Injury Network composed of Nephrologist and Intensivist modified the Rifle criteria and defined AKI as an abrubt (within 48 hours) reduction in kidney function i.e. absolute increase in serum creatinine of more than or equal to $0.3 \mathrm{mg} / \mathrm{dl}$, increase in serum creatinine of more than equal to $50 \%$ or a reduction in urine output (documented oliguria of $<$ than 0.5 $\mathrm{ml} / \mathrm{kg} / \mathrm{hr}$ for more than 6 hours) .5

Total number of patients who fulfilled the inclusion criteria were 30. Exclusion criteria were Chronic Kidney Disease who were on RRT (renal replacement therapy) and who didn't give the consent. All the patient with Standard demographic, clinical and physiological were studied in detail. Demographic study included age, sex, gender, ethnicity, and duration of ICU stay. Clinical data included primary diagnosis, presence of comorbid conditions, laboratory investigations, treatment history, and need for renal replacement therapy. Data on kidney function included serum creatinine, urea, sodium, potassium, urine output, metabolic profile and hematological profile.

We defined diagnostic category on the basis of documentation during the admission by a treating Physician. The primary reason for sepsis and septic shock were made and patients admitted with sepsis related secondary to Pneumonia, Acute gastroenteritis, Urinary Tract Infections, soft tissue infections and sepsis of Undetermined source. Follow up data regarding their renal function status was collected. Statistical calculations were done by SPSS version (statistical package for the social sciences). Ethical clearance was obtained from the Ethics Review Committee. Informed written consent was obtained from patients, or from the closest relative. All investigations that the patients were subjected to were a part of the routine workup done in any critically ill patient. No personal information was collected.

\section{RESULTS}

A total of 30 patents admitted in ICU who met the inclusion criteria were studied. Out of 30 cases 19 were males and 11 were females. Maximum number of patents were in between $51-60$ years (33.3\%). According to sex, out of 30 , nineteen $(63.3 \%)$ were male and eleven (36.7\%) were female.

Table 1: Age distribution of patients

\begin{tabular}{|l|l|l|}
\hline Age & $\begin{array}{l}\text { Number of } \\
\text { patients }\end{array}$ & Percentage \\
\hline $18-30$ & 2 & 6.7 \\
\hline $31-40$ & 3 & 10 \\
\hline $41-50$ & 2 & 6.7 \\
\hline $51-60$ & 10 & 33.3 \\
\hline $61-70$ & 7 & 23.3 \\
\hline $71-80$ & 4 & 13.3 \\
\hline
\end{tabular}

In this study ten out of thirty patients were inthe age group between $51-60$ years i.e $33.3 \%$. 7 patients $(23.3 \%)$ were in age group 61-70.

Table 2: Ethnicity

\begin{tabular}{|l|l|l|}
\hline Ethinicity & Frequency & Percentage \\
\hline Brahmin & 6 & 20 \\
\hline Chhetri & 7 & 23.3 \\
\hline Mongol & 9 & 30 \\
\hline MADHESI & 2 & 6.7 \\
\hline
\end{tabular}




\begin{tabular}{|l|l|l|}
\hline OTHERS & 6 & 20 \\
\hline TOTAL & 30 & 100 \\
\hline
\end{tabular}

Among the thirty patients, the maximum belonged to the Mongolian group i.e. $30 \%$ followed by Brahmin and chhetris and the lowest being the madhesis i.e. $6.7 \%$.

Table 3: Causes Of AKI

\begin{tabular}{|l|l|l|}
\hline Diagnosis & Frequency & Percentage \\
\hline Sepsis & 14 & 46.6 \\
\hline Gastroenteritis & 03 & 10.0 \\
\hline Hepatic & 05 & 16.6 \\
\hline Cardiac & 01 & 3.4 \\
\hline Surgical & 02 & 6.6 \\
\hline Obstetrical & 02 & 6.6 \\
\hline Others & 03 & 10.2 \\
\hline Total & 30 & 100 \\
\hline
\end{tabular}

Sepsis was the most common cause of AKI among the patients admitted in icu. Fourteen out of thirty i.e around $47 \%$ of AKI was due to sepsis. The other causes of AKI were hepatic (alcoholic hepatitis), gastroenteritis, postoperative cases, obstetric cases and one of them was due to cardiac case respectively.

Table 4: RIFLE CRITERIA

\begin{tabular}{|l|l|l|}
\hline RIFLE & Frequency & Percentage \\
\hline Risk & 04 & 13.3 \\
\hline Injury & 16 & 53.3 \\
\hline Failure & 07 & 23.3 \\
\hline Loss & 03 & 10.0 \\
\hline Total & 30 & 100 \\
\hline
\end{tabular}

According to the RIFLE criteria more than $50 \%$ of patients fall in Injury group, which was followed by failure and risk. Only $10 \%$ of the cases were in loss category.

Table 5: RIFLE criteria and outcome

\begin{tabular}{|l|l|l|l|}
\hline RIFLE & \multicolumn{2}{|c|}{ Outcome } & \\
\hline & Recovery & Death & Total \\
\hline Risk & 03 & $01(14.2 \%)$ & 04 \\
\hline Injury & 10 & $06(37.5 \%)$ & 16 \\
\hline Failure & 04 & $03(42.8 \%)$ & 07 \\
\hline Loss & 02 & $01(33.3 \%)$ & 03 \\
\hline Total & 19 & 11 & 30 \\
\hline
\end{tabular}

According to Rifle Criteria, 10 out of 30 patients fell in injury group and six patients from the same group died.

\section{Table 6: Need of RRT}

\begin{tabular}{|l|l|l|l|}
\hline \multirow{2}{*}{$\begin{array}{l}\text { Need of } \\
\text { RRT }\end{array}$} & \multicolumn{2}{|c|}{ Outcome } & \multirow{2}{*}{ Total } \\
\cline { 2 - 3 } & Recovery & Death & \\
\hline Yes & 03 & 05 & $8(26.7 \%)$ \\
\hline No & $16(84.21 \%)$ & 6 & $22(73.3 \%)$ \\
\hline Total & $19(63.3 \%)$ & $11(36.7 \%)$ & 30 \\
\hline
\end{tabular}

In this study of 30 cases, 19(63.3\%) patients survived and about 11 (36\%) died. 8 patients (26.7\%) needed RRT out of which only 3 survived and 5 died. However, 22 patients did not undergo any RRT, 16 patients survived with conservative management whereas 6 patients died before undergoing any RRT.

\section{DISCUSSION}

AKI is one of the most common syndrome encountered in ICU. In our study of 30 patients, nineteen (63.3\%) were males and eleven (36.6\%) were female which is similar to the studies done by Chettri et al.5 Most of AKI patients were from age group of 51- 60 years (33.3\%). In contrast to our study Cystowski et al. showed that most of AKI occur above the age of 65 years and has higher burden of illness. ${ }^{6}$ We also tried to compare which of the ethnic groups suffered from AKI and we found Mongol group to be mostly affected. However, no such studies on ethnic groups in our country are available till date.

The most common cause of AKI in our study was sepsis which was followed by other medical causes such as gastroenteritis, hepatic and cardiac cases. There were few surgical and obstetrics cases too. Eswarappa et al who evaluated acute kidney injury in tertiary centre in South India found sepsis to be the commonest cause of acute kidney injury. Shende et al also found that sepsis to be the most common cause of AKI admitted in ICU. ${ }^{6}$ However in contrast to our studies Ghimire et al7 concluded pneumonia to be the commonest cause of AKI admitted in tertiary centre ICU. A study conducted by Khakurel et al. found gastroenteritis to be the most common cause of AKI in Nepal. ${ }^{10}$

In this study most of the patients fell into injury group comprising maximum deaths and recovery in same group. The hospital mortality rate was $14.2 \%$ for risk 
patient, $36 \%$ injury, $42 \%$ in failure and $33 \%$ in loss patient similar to the mortality study conducted by Park et al (29\% for risk, 36\% for injury, 51\% for failure patients)..$^{11}$

Out of total 30 patients admitted in ICU, nineteen (63.3\%) survived and eleven (36.7\%) died. Only 3 patients who survived needed renal replacement therapy, however5outof6patientwhodiedunderwent renal replacement therapy. In contrast the studies conducted by Chiioke et al found mortality (46.6\%) higher than the studies conducted in our hospital. ${ }^{8}$ The main reason behind RRT were multifactorial, the most common cause being decreased urine output followed by refractory hyperkalemia and metabolic acidosis. Studies conducted by Daniel et al found metabolic acidosis to be the most common indication for RRT.9 The research conducted by Daniel et al9 had higher renal recovery rate (80\%) as compared to our studies (63.3\%).

\section{CONCLUSION}

The results in this study show that AKI patients can be treated without doing RRT if patients conditions are detected early with judicial investigations. However late stages in AKI have poorer prognosis even with RRT. Our studies showed sepsis to be the most common cause of AKI admitted in ICU.

\section{REFERENCES}

1. Kashinkunti MD etal. Clinical spectrum of acute kidney injury: a study from tertiary care hospital. International Journal of Pharmaceutical and Biological Research. 2013; 04; 165-169.

2. Hady HA, Baghdadi IM, Kora MA. Acute kidney injury outcome in critically ill patients: International Journal of Current Research. 2014; 02; 5018-5025.

3. Shende P, Shara SK, Vikhe VB. Clinical profile of acute kidney injury in intensive care unit in tertiary care centre: Indian Journal of Basic and Applied Medical research. 2014; 02; 511-516.

4. Eswarappa M, Gireesh MS, Kumar D. Spectrum of acute kidney injury in critically ill patients: a single center study from South India. Indian Journal Of Nephrology. 2014; 24; 280-284.
5. Chhetri PK, Manandhar DN, Pahari LR. Acute renal failure in Nepal Medical College Teaching Hospital. Nepal Medical College Journal. 2008; 10; 132-135.

6. Czystowski M, Daniewska D, Gellert R. acute renal injury incidence is growing in the elderly inpatients-a tertiary level academic single centre observation over 5 consecutive years. Postepy Nauk Medycznych. 2013; 02; 125-126.

7. Ghimire M, Pahari B, Sharma SK. Outcome of sepsis associated acute kidney injury in an intensive care unit: an experience from tertiary care centre of central Nepal. Saudi Journal of Kidney Diseases and transplantation. 2014; 25; 912-917.

8. Chijioke A, Makusidi AM. Severe acute kidney injury in adult Nigerians from University of Ilorin teaching hospital. Borno Medical Journal. 2011; $8 ; 1-5$.

9. Franzen D, Rupprecht C, Hauri D, Bleisch JA. Predicting outcome in critically ill patients with acute kidney injury undergoing intermittent hemodialysis- a retrospective cohort analysis. International Journal Artificial Organs. 2010; 33; 15-21.

10. Khakurel S, Satyal PR, Agarwal RK, Chhetri PK. Acute renal failure in a tertiary care center in Nepal. Journal of Nepal Medical Association. 2005; 44; 32-35.

11. Park WY, Hwang EA, Jang MH. The risk factors and outcome of acute kidney injury in the intensive care units. The Korean Journal of Internal Medicine. 2010; 25; 181-187. 